\title{
Avaliação do sistema de vigilância das intoxicações exógenas no âmbito da saúde do trabalhador no Brasil entre 2007 e 2009
}

\author{
Evaluation of the Brazilian national epidemiological surveillance system \\ for exogenic poisoning in the occupational health from 2007 to 2009
}

Hiane Santos de Jesus ${ }^{1}$, Henrique de Barros Moreira Beltrão², Dalva Maria de Assis ${ }^{3}$

\begin{abstract}
Resumo
O sistema de vigilância deve ser avaliado periodicamente, visando tornar mais efetivas e ágeis as medidas de controle de eventos adversos à saúde. Dessa forma, os objetivos desse estudo foram avaliar os atributos qualitativos e quantitativos do sistema de vigilância das intoxicações exógenas do Brasil de 2007 a 2009, período em que este se vinculava exclusivamente à Saúde do Trabalhador, por meio do Update Guidelines for Evaluating Public Health Surveillance Systems. Foi utilizado um formulário estruturado e o banco de dados do SINAN NET como fontes de dados. Dos 27 estados do Brasil, 16 responderam aos questionários. Os dados seguem o fluxo estabelecido pelo SINAN NET sem a definição de uma coordenadoria estadual. Conforme os parâmetros adotados, esse sistema de vigilância foi considerado simples, flexível, oportuno e instável. A qualidade de dados e a representatividade foram regulares; e a sensibilidade e o valor preditivo positivo foram baixos. Recomendamos estabelecer o fluxo do sistema de vigilância dentro das secretarias estaduais e elaborar guia das intoxicações exógenas, definindo os objetivos para esta vigilância.
\end{abstract}

Palavras-chave: vigilância epidemiológica; saúde do trabalhador; envenenamento.

\begin{abstract}
The surveillance systems should be evaluated periodically with objective to become more agile and effective measures to control adverse health events. Thus, the aim of this study was to evaluate the qualitative and quantitative attributes of the Surveillance System for Exogenic Poisoning in Brazil from 2007 to 2009, during which it was linked exclusively to the Occupational Health. For this purpose, there was used the Update Guidelines for Evaluating Public Health Surveillance Systems. A structured questionnaires and database SINAN NET was used as data sources. Of the 27 states of Brazil, 16 answered the questionnaires. The data follow the flow established by Brazilian Surveillance System (SINAN NET), however without setting a statewide coordinating body. As the parameters adopted, the Surveillance System for Exogenic Poisoning was considered simple, flexible, timely and unstable. Data quality and representativeness were regular; and the sensitivity and Positive Predictive Value were low. We recommend establishing a Surveillance System's flow within the state and to develop a Guide for Exogenic Poisoning, defining the goals for this surveillance.
\end{abstract}

Keywords: epidemiological surveillance; occupational health; poisoning.

Trabalho realizado na Secretaria de Vigilância em Saúde (SVS), Ministério da Saúde (MS) - Brasil (DF), Brasil.

'Especialista em Saúde da Família, em Saúde Coletiva e em Enfermagem do Trabalho; Enfermeira de Programa de Saúde da Família (PSF) vinculada à

Secretaria Municipal de Saúde de Aracaju - Aracaju (SE), Brasil; Treinanda do Programa de Treinamento em Epidemiologia Aplicada aos Serviços do Sistema Único de Saúde (EPISUS), Coordenação Geral de Vigilância e Resposta às Emergências em Saúde Pública (CGVR), Departamento de Vigilância das Doenças Transmissíveis (DEVEP), Secretaria de Vigilância em Saúde (SVS), Ministério da Saúde (MS) - Brasília (DF), Brasil.

2Mestre em Saúde Pública. Monitor do Programa de Treinamento EPISUS, CGVR, DEVEP, SVS, MS - Brasília (DF), Brasil.

${ }^{3}$ Mestranda em Avaliação e Monitoramento em Saúde Pública. Supervisora do Programa de Treinamento EPISUS, CGVR, DEVEP, SVS, MS - Brasília (DF), Brasil. Endereço para correspondência: Hiane Santos de Jesus - Avenida Adélia Franco, 2.750 - Condomínio Residencial Portucale, Edifício Porto, apto 1.004 CEP: 49048-010 - Aracaju (SE), Brasil - E-mail: hianesj@hotmail.com

Fonte de financiamento: Conselho Nacional de Desenvolvimento Científico e Tecnológico (CNPq).

Conflito de interesse: nada a declarar. 


\section{INTRODUÇÃO}

Intoxicação é a manifestação, através de sinais e sintomas, dos efeitos nocivos produzidos em um organismo vivo como resultado da sua interação com alguma substância química (exógena) ${ }^{1}$. As substâncias químicas podem ser encontradas no ambiente (ar, água, alimentos, plantas, animais peçonhentos ou venenosos etc.) ou isoladas (pesticidas, medicamentos, produtos de uso industrial, produtos de uso domiciliar etc.) $)^{2}$. Segundo a Organização Mundial de Saúde (OMS), as intoxicações, acidentais ou intencionais são importantes causas de agravos à saúde ${ }^{1}$. Estima-se que 1,5 a $3 \%$ da população intoxicam-se todos os anos ${ }^{1}$. Para o Brasil, isto representa aproximadamente 4.800 .000 casos novos a cada ano, destes, 0,1 a $0,4 \%$ das intoxicações resultam em óbito ${ }^{1}$.

A notificação das Intoxicações Exógenas pelo Sistema de Informação de Agravos de Notificação (SINAN) iniciou-se em 1997, quando a Portaria $n^{\circ} 168$ inseriu a intoxicação por agrotóxicos na Lista Nacional das Doenças de Notificação Compulsória. Nesse período, o sistema era vinculado à Divisão de Meio Ambiente e Ecologia Humana ${ }^{3}$. Posteriormente, foram promulgadas as Portarias $n^{\circ} 2.325 / 2003$ e $33 / 2005$, porém estas não mantinham as intoxicações por agrotóxicos nesta lista ${ }^{4,5}$.

Em 2004, a Portaria $n^{\circ} 777$ estabeleceu que as intoxicações exógenas (por substâncias químicas, incluindo agrotóxicos, gases tóxicos e metais pesados), a partir daquele momento, deveriam ser tratadas como um agravo à saúde do trabalhador de notificação compulsória, sendo registrados no SINANNET . Este componente da vigilância das intoxicações exógenas fornece as informações tanto para o acompanhamento dos casos individualmente, como para a identificação do perfil epidemiológico desses trabalhadores. Para esse fim, as unidades de notificação seriam as redes de serviços sentinelas especificamente ligadas à Rede Nacional de Atenção Integral à Saúde do Trabalhador (Renast) e vinculadas à Coordenação Geral de Saúde do Trabalhador ${ }^{6}$. A Renast é um modelo de atenção integral à saúde dos trabalhadores, cuja rede sentinela é composta por serviços médicos e ambulatoriais de média e alta complexidade responsáveis por diagnosticar os acidentes e doenças relacionados ao trabalho, por registrálos no SINAN NET e emitir a Comunicação de Acidente de Trabalho (CAT) ${ }^{7}$.

Vale ressaltar que os sistemas de vigilância devem ser avaliados periodicamente e, se necessário, alterados de maneira a garantir seu bom desempenho, adequando-os as modificações da estrutura, grau de desenvolvimento e complexidade tecnológica do Sistema Nacional de Saúde ${ }^{8,9}$. Esse aprimoramento dos sistemas de vigilância visa tornar mais efetivas e ágeis as medidas de controle de eventos adversos à saúde ${ }^{8}$.
Dessa forma, esse é o primeiro estudo voltado para a avaliação do sistema de vigilância epidemiológica das intoxicações exógenas, apoiado no sistema de informação nacional, no âmbito da Saúde do Trabalhador; e teve o intuito de avaliar os atributos qualitativos e quantitativos desse sistema no Brasil entre 2007 e 2009, com vistas à melhoria do mesmo.

\section{METODOLOGIA}

A avaliação do sistema de vigilância foi baseada nas diretrizes do Update Guidelines for Evaluating Public Health Surveillance Systems, contemplando a avaliação dos atributos qualitativos e quantitativos do sistema de vigilância das intoxicações exógenas ${ }^{10}$.

As fontes de informação utilizadas foram o banco de dados do SINAN NET das Intoxicações Exógenas no período de 2007 a 2009, dados congelados até junho de 2010. Destacamos que o sistema de informação da vigilância das intoxicações exógenas foi criado em ambiente Windows e implantado em 2006, apesar de utilizar algumas variáveis do sistema de informação da vigilância das intoxicações por agrotóxicos da versão em ambiente DOS. Além dessa fonte, foram utilizados os formulários enviados por meio eletrônico (e-mail) através do FormSUS para 14 técnicos do nível central responsáveis pela vigilância das Intoxicações Exógenas, e 54 coordenadores estaduais, sendo 27 da Saúde Ambiental e 27 da Saúde do Trabalhador.

Para avaliar o fluxo entre nível estadual e municipal, utilizamos como referência as informações do estado Paraná e de sua capital Curitiba. Esses locais foram eleitos por serem responsáveis pela maior parte das notificações no período analisado.

Os softwares utilizados foram Epi-Info versão 3.5.3, TabWin versão 3.2, RecLink III e Microsoft Excel 2010.

\section{Critérios para avaliação dos atributos qualitativos}

A simplicidade refere-se à facilidade de operação do sistema em suas etapas ${ }^{10}$. Para avaliá-la utilizou-se a opinião dos técnicos estaduais quanto ao número de variáveis da Ficha Individual de Investigação (FII), ao grau de complexidade para obtenção das informações da FII, às dificuldades na utilização do SINAN NET, ao tempo gasto para entrar, editar e armazenar dados da FII, além do fluxo percorrido desde a coleta dos dados frente a um caso suspeito até a sua chegada ao Ministério da Saúde.

A flexibilidade é a habilidade do sistema para adaptar-se às mudanças ${ }^{10}$. Assim, a flexibilidade do sistema foi avaliada sobre a ótica dos tipos de unidades notificadoras no período estudado. Essas unidades podem ser classificadas quanto à 
tipologia em hospitais, pronto atendimento, unidades de saúde da família ou de atenção básica à saúde, entre outras.

A qualidade dos dados reflete o nível de completitude e validade dos dados registrados ${ }^{10}$. A completitude foi calculada através do percentual de campos ignorados ou em branco, sendo classificada em excelente quando o percentual fosse superior a $90 \%$, regular de 70 a $89 \%$, e ruim quando abaixo de $70 \%{ }^{11}$. Também foi observado o número de registros duplicados, para o qual se utilizou o método probabilístico através do software Reclink III. As variáveis de blocagem foram nome do paciente, sexo e município de residência e as variáveis de comparação foram nome do paciente, nome da mãe, data de nascimento e data dos primeiros sintomas. O SINAN NET estabelece que um banco de dados com até $5 \%$ de duplicidade é considerado satisfatório, sendo este o parâmetro adotado ${ }^{11}$. E, por fim, foi avaliada a consistência dos dados compreendida como a coerência entre as categorias assinaladas entre dois campos relacionados ${ }^{10}$, para tal confrontaram-se as seguintes variáveis: "exposição/contaminação decorrente de trabalho" e "CAT", "tipo de atendimento" e "primeiros sintomas". Os parâmetros adotados para avaliar as inconsistências foram: abaixo de $10 \%$ considerou-se excelente, de 10 a $30 \%$ regular e acima de $30 \%$ ruim? .

A aceitabilidade reflete a vontade das pessoas e instituições em participarem do sistema de vigilância ${ }^{10}$. Este atributo foi avaliado a partir da utilização de sistemas paralelos; qualidade dos dados; prestação de assessorias técnicas; produção de boletins epidemiológicos e material educativo.

\section{Critérios para avaliação dos atributos quantitativos}

A sensibilidade de um sistema refere-se à proporção de casos detectados no sistema avaliado em relação a outro sistema considerado padrão ouro ${ }^{10}$. Optou-se por calcular a sensibilidade em relação ao Sistema de Informação sobre Mortalidade (SIM), uma vez que todo óbito de intoxicação exógena, a princípio, deve estar presente nesses dois bancos.

Para tanto, foram feitos dois recortes: faixa etária, de 20 a 65 anos e Classificação Estatística Internacional de Doenças e Problemas Relacionados à Saúde (CID-10) do capítulo XIX sobre lesões, envenenamento e algumas outras consequências de causas externas (S00-T98) e do capítulo XX das causas externas de morbidade e de mortalidade (V01-Y98) ${ }^{12-14}$, como observado abaixo:

- T36-T50 - intoxicação por drogas, medicamentos e substâncias biológicas;

- T51-T65 - efeitos tóxicos de substâncias de origem predominantemente não medicinal;

- X40-X49 - envenenamento [intoxicação] acidental por e exposição a substâncias nocivas;
- X60-X69 - lesões autoprovocadas intencionalmente;

- X85-X90 - agressões;

- Y10-Y19 - eventos (fatos) cuja intenção é indeterminada.

A taxa de desemprego entre 2007-2009, calculada através da Pesquisa Mensal de Desemprego ${ }^{15}$, foi utilizada como tentativa de controlar os denominadores, visto que o SIM não é restrito apenas à população economicamente ativa (PEA) e o SINAN NET, no período analisado, priorizava esta população. Desta forma, tem-se que a sensibilidade (Se) é igual ao número de óbitos registrados no SINAN NET dividido pelo número de óbitos registrados no SIM, descontada a taxa de desemprego do país no período multiplicado por 100.

O valor preditivo positivo (VPP) reflete a proporção dos casos confirmados em relação ao total de casos notificados no sistema ${ }^{10}$. Esse foi calculado dividindo-se o número de casos confirmados por qualquer critério de confirmação, com descrição do agente tóxico diferente de ignorado ou em branco, pelo número de casos notificados no sistema multiplicado por 100.

Um sistema deinformaçãoem vigilânciaédito representativo quando é capaz de descrever com boa precisão a ocorrência dos casos por pessoa, tempo e lugar ${ }^{10}$. Apesar das notificações dos casos de intoxicações exógenas estarem restritas à Saúde do Trabalhador no período avaliado, percebeu-se uma diversidade de outras situações não relacionadas ao trabalho, tais como acidentes na infância (19.465 notificações) e tentativas de suicídio (32.319 notificações). Optou-se então, por avaliar a representatividade através da análise dos acidentes de trabalho (6.953 notificações) ocorridos por intoxicação exógena por agrotóxicos de uso agrícola, devido à disponibilidade de informações na literatura. As lavouras de fumo e milho foram eleitas para comparação entre estas e os casos de intoxicações exógenas por agrotóxicos de uso agrícola, pois foram as mais frequentemente relatadas no banco do SINAN NET com ocorrências de 19 e $11 \%$, respectivamente.

A oportunidade do sistema mede a velocidade entre dois passos do sistema de vigilância ${ }^{10}$. Dessa forma, foram calculadas a oportunidade de atendimento medida entre o tempo de exposição e atendimento; a oportunidade de notificação, medida entre o início dos sintomas e a notificação; a oportunidade de investigação, medida entre notificação e investigação; e a oportunidade de encerramento de caso, medida entre a notificação e encerramento da investigação. Segundo a Portaria $\mathrm{n}^{\circ} 104$ de 25 de janeiro de 2011, os agravos referentes às intoxicações exógenas estão incluídos nos anexos 1 e 2, portanto, devem ser notificados em até 24 horas $^{16}$. A investigação dos casos suspeitos deve ocorrer em até 24 horas e o encerramento da investigação epidemiológica em até 60 dias ${ }^{11}$. Arbitrou-se que variáveis com percentual de oportunidade 
superior ou igual a $70 \%$ fossem consideradas adequadas, e se inferior a 70\% consideradas inadequadas.

A estabilidade do sistema reflete a confiança e a disponibilidade do sistema de informação ${ }^{10}$. Essa foi calculada a partir da concepção dos técnicos quanto à disponibilidade do sistema operacional; do quantitativo de recursos materiais e humanos envolvidos nessa vigilância, que poderiam ser classificados desde muito insuficiente até em excesso, e o tempo em que estes técnicos estão desenvolvendo atividades nessa área.

\section{RESULTADOS}

Essas análises foram embasadas por 5 (39\%) formulários respondidos pelos técnicos do nível central, e por 16 (59\%) formulários provenientes dos estados que atenderam a demanda. O banco de dados do SINAN NET utilizado para as análises continha 96.048 registros de intoxicações exógenas no Brasil entre 2007 e 2009, porém apenas 6.953 notificações eram vinculadas aos acidentes de trabalho. Vale ressaltar que em algumas análises fez-se esse recorte, visto que se trata do foco do presente estudo.

O estudo identificou que, em 14 estados mais o Distrito Federal, o sistema de vigilância das intoxicações exógenas ficou sob a responsabilidade da Vigilância Ambiental; em outros 10 estados a responsabilidade coube à Coordenação de Saúde do Trabalhador ou ao Centro de Referência de Saúde do Trabalhador Estadual (CEREST); e, em outros 2 estados essa vigilância coube à Coordenação das Doenças Não Transmissíveis ou à Vigilância Epidemiológica Estadual distintamente (Figura 1).

\section{Atributos qualitativos \\ Simplicidade}

O número de variáveis da FII foi considerado suficiente por $3(60 \%)$ técnicos do nível central e $11(79 \%)$ técnicos estaduais. Vale ressaltar que, atualmente, a FII é composta por quatro grupos: antecedentes epidemiológicos, dados da exposição, dados do atendimento e conclusão do caso. São 77 variáveis, das quais apenas a variável "evolução do caso" não pode ser preenchida no primeiro atendimento. Nessa FII não foram contempladas as informações referentes ao quadro clínico do paciente e os dados laboratoriais. Quanto à complexidade necessária para a obtenção das informações da FII, 11 (73\%) técnicos do nível estadual consideraram-na simples. Não foram relatadas dificuldades para utilização do SINAN NET por $3(60 \%)$ técnicos do nível central e por $12(75 \%)$ do nível estadual; apesar de observamos ausência de conceitos formais básicos a cerca da vigilância das intoxicações exógenas, existindo formalizada apenas a definição de caso suspeito de intoxicação exógena, presente no cabeçalho da FII. Outras definições como caso confirmado ou descartado de intoxicação exógena, bem como os critérios de confirmação laboratorial e os objetivos do sistema não possuem registros oficiais. Para entrada, edição e armazenamento dos dados os técnicos relataram um tempo mediano de 15 minutos (3 minutos-30 dias).

Em relação ao fluxo do sistema, os técnicos do nível central e estadual entrevistados informaram que, inicialmente, há o preenchimento da Ficha de Notificação Individual (FNI) e esta, por sua vez, gera a investigação do caso suspeito com o posterior preenchimento da FII pela unidade notificadora. Em seguida, essas fichas são encaminhadas para as Secretarias Municipais de Saúde que consolidam os dados das unidades notificadoras e, semanalmente, enviam os dados para as Secretarias Estaduais através do SISNET, e-mail ou disquete. Estas consolidam as informações dos municípios e encaminham-nas, quinzenalmente, para o Ministério da Saúde através do SISNET. No Ministério da Saúde, esses dados são enviados para o Departamento de Vigilância em Saúde Ambiental e Saúde do Trabalhador para monitoramento.

\section{Flexibilidade}

Esse atributo foi avaliado pela identificação dos tipos de unidades notificadoras no período, uma vez que essas notificações só deveriam ocorrer nos serviços sentinelas da Renast $^{6}$. No entanto, foi observado que as notificações foram realizadas pela Vigilância Epidemiológica, quando esta fora acionada via estabelecimentos de indústria, agricultura e pecuária, bem como outros serviços de saúde pertencentes à atenção básica e à rede de urgência e emergência.

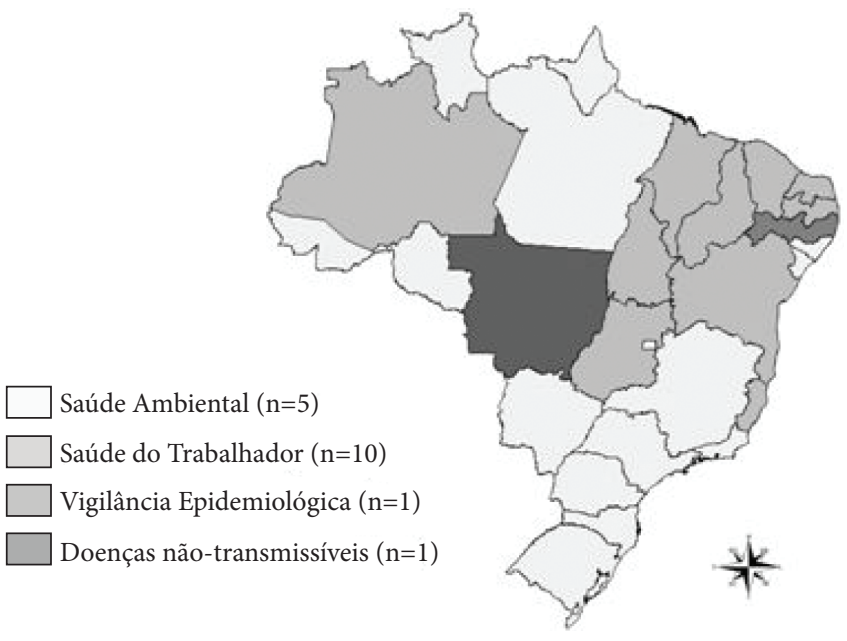

Figura 1. Distribuição das coordenações estaduais das intoxicações exógenas, Brasil, 2007-2009 


\section{Qualidade dos dados}

Verificou-se que, dos 96.048 registros de intoxicações exógenas ocorridos entre 2007-2009, foram identificadas 3.012 (3\%) duplicidades. Com relação ao percentual de completitude das variáveis da FII, identificou-se uma média de $69 \%$ de campos preenchidos. No cruzamento das informações referentes às variáveis "emissão da CAT" e "exposição/contaminação decorrente de trabalho", tem-se que, em 417 (2\%) casos houve emissão de CAT, porém sem caracterização de acidente de trabalho, o que configura uma inconsistência do sistema, assim como, em 3.024 (79\%) acidentes de trabalho não ocorreu essa emissão. Quanto ao tipo de exposição, observou-se que, em 6.860 (16\%) exposições agudas, os primeiros sintomas ocorreram num período superior a 48 horas, o que é uma inconsistência, bem como 525 (81\%) exposições crônicas ocorrerem num intervalo de tempo menor ou igual há 48 horas.

\section{Aceitabilidade}

A utilização de sistemas paralelos para coleta e sistematização das informações sobre as intoxicações exógenas foi relatada por 3 (27\%) técnicos estaduais. A prestação de assessoria técnica foi informada por 2 (40\%) técnicos do Ministério da Saúde e 5 (33\%) técnicos estaduais. Segundo os entrevistados, 1 (7\%) estado elaborou boletim epidemiológico no período e 7 (44\%) elaboraram material educativo. Não houve relato de confecção de boletins epidemiológicos ou materiais educativos pelo nível central.

\section{Atributos quantitativos}

\section{Sensibilidade}

A sensibilidade de captação de óbitos desse sistema para o país foi de 18\% em 2007, 21\% em 2008 e 24\% em 2009, sendo que a sensibilidade média para o período foi de $21 \%$, conforme cálculos abaixo:

$$
\begin{aligned}
& \mathrm{Se}_{2007}=\frac{292}{1.814(1-0,093)} \times 100=18 \% \\
& \mathrm{Se}_{2008}=\frac{352}{1.854(1-0,078)} \times 100=21 \% \\
& \mathrm{Se}_{2009}=\frac{379}{1.716(1-0,081)} \times 100=24 \% \\
& \mathrm{Se}_{2007-2009}=\frac{18+21+23}{1.814(1-0,093)}=21 \%
\end{aligned}
$$

\section{Valor preditivo positivo}

O VPP do sistema no período foi de $60 \%$, como se visualiza através do cálculo seguinte.
$\mathrm{VPP}_{2007-2009}=\frac{55.589}{93.036} \times 100=60 \%$

\section{Representatividade}

O artigo de Bedor et al. ${ }^{17}$ sobre "Vulnerabilidades e situações de riscos relacionados ao uso de agrotóxicos na fruticultura irrigada" envolveu 283 sujeitos do estudo com o objetivo de conhecer o contexto social, econômico e cultural e algumas das vulnerabilidades para a saúde relacionadas com o processo produtivo químico-dependente da fruticultura do Vale do São Francisco, para assim contribuir com ações de proteção da saúde dos trabalhadores rurais frente ao uso de agrotóxicos em um modelo produtivo agro-exportador. Ao avaliar os dados do SINAN NET da região com o trabalho de Bedor et al ${ }^{17}$, observa-se que há diferença estatisticamente significativa entre as proporções de características como sexo e escolaridade, enquanto que as proporções referentes à situação no mercado de trabalho e idade mostraram-se semelhantes (Tabela 1).

Os dados sobre os casos de intoxicações exógenas por agrotóxicos de uso agrícola e das lavouras aonde esses produtos foram utilizados demonstraram que as mudanças nos processos de trabalho acompanharam a frequência de surgimento de casos nas lavouras de fumo e milho. Na lavoura do fumo, observa-se que o maior número de intoxicações acontece na atividade de colheita, e, portanto entre os meses de novembro e fevereiro (Figura 2).

$\mathrm{Na}$ lavoura do milho, percebe-se que 91 (39\%) casos de intoxicação exógena são decorrentes do tratamento das sementes, seguidos de $85(36 \%)$ casos devido à pulverização da lavoura. Observa-se o aumento do número de casos de intoxicações por agrotóxicos no decorrer dos anos, principalmente, no que se refere ao tratamento de sementes, entre os meses de janeiro e abril. Ainda, percebe-se que a pulverização apresentou um incremento nos meses de dezembro a abril, sendo que, em 2009, esse aumento de intoxicações exógenas foi observado entre outubro e novembro (Figura 3). Outro

Tabela 1. Características de trabalhadores intoxicados por agrotóxicos, Vale Médio do São Francisco (PE-BA), 2007-2009

$\begin{array}{lccc}\text { Características n (\%) } & \begin{array}{c}\text { Bedor et al }^{17} \\ \mathbf{n = 2 8 3}\end{array} & \begin{array}{c}\text { SINAN NET } \\ \mathbf{n = 6 3}\end{array} & \text { Valor } \mathbf{p}^{*} \\ \begin{array}{l}\text { Sexo masculino } \\ \begin{array}{l}\text { Escolaridade até } \\ \text { primeiro grau }\end{array}\end{array} & 234(83) & 42(67) & <0,01 \\ \begin{array}{l}\text { Empregado com } \\ \text { carteira assinada }\end{array} & 155(55) & 22(36) & 0,46 \\ \begin{array}{l}\text { Idade (média em anos) } \\ \text { *teste do } \chi^{2} \text {; DP: desvio padrão }\end{array} & 35(\mathrm{DP}=12) & 31(\mathrm{DP}=14) & \\ \begin{array}{l}\text { Fonte: SINAN NET, Brasil, 2007-2009 } \\ \end{array} & & \end{array}$




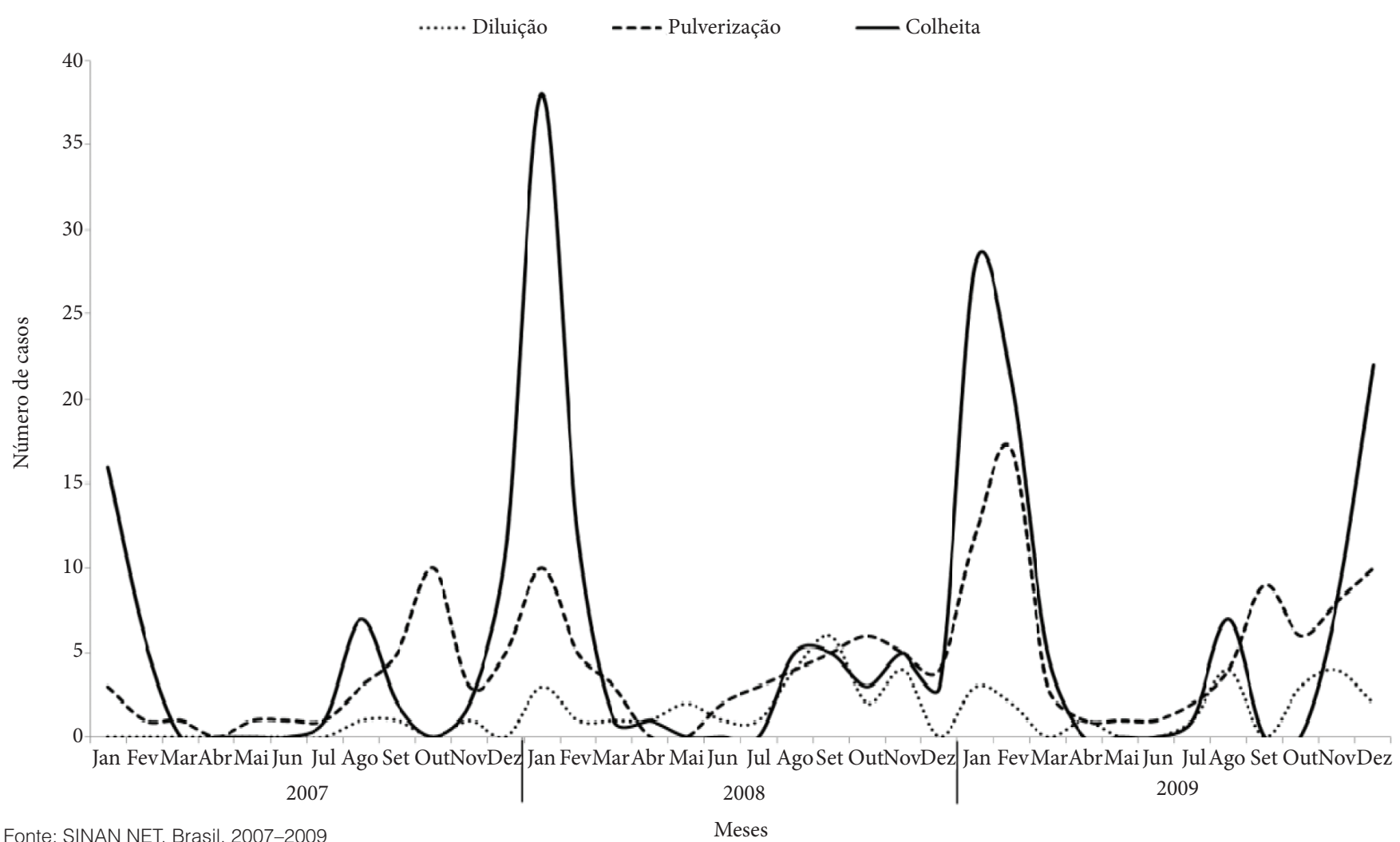

Figura 2. Distribuição temporal dos casos de intoxicações por agrotóxicos segundo atividade desenvolvida pelo trabalhador na lavoura de fumo, Brasil, 2007-2009

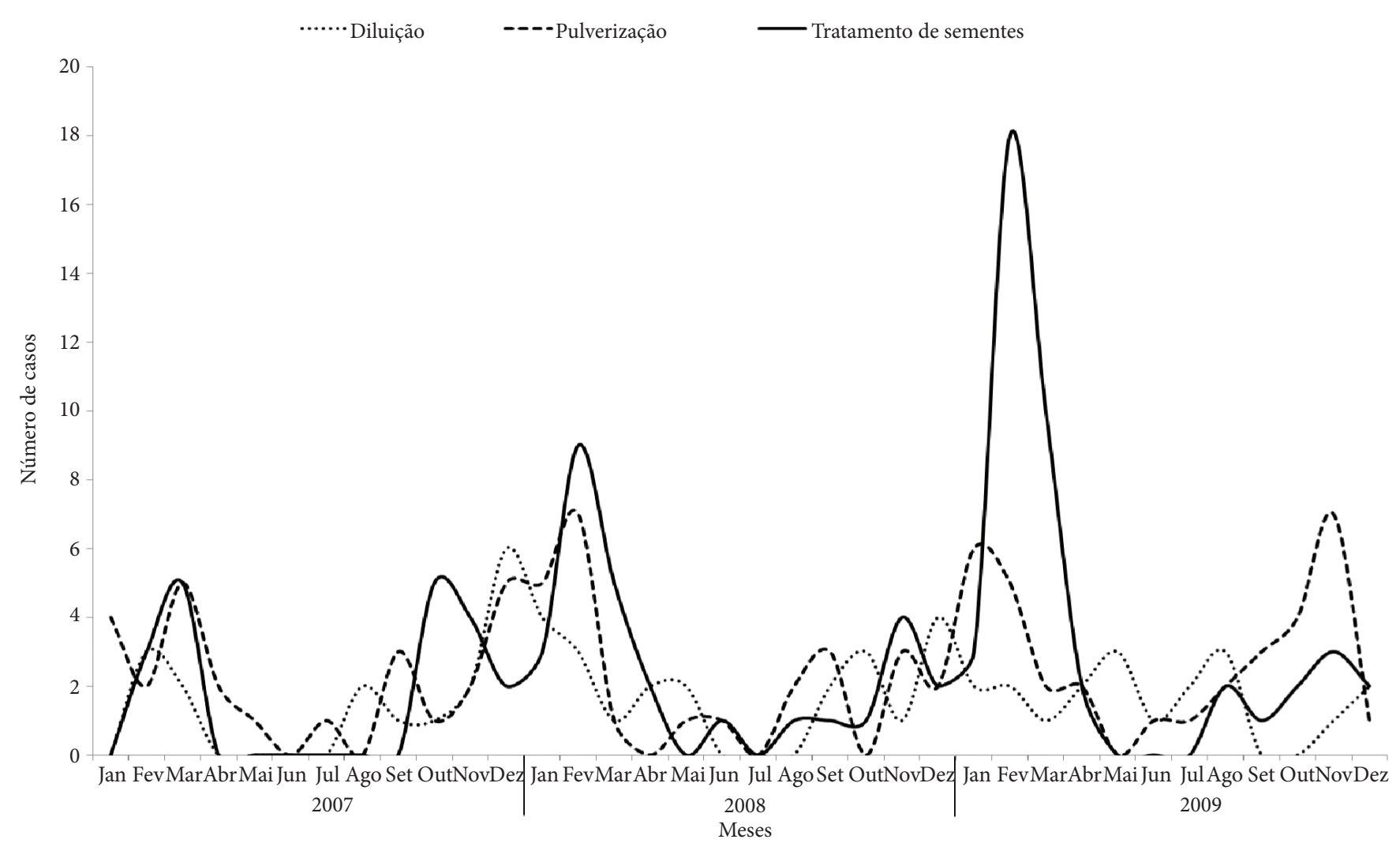

Fonte: SINAN NET, Brasil, 2007-2009

Figura 3. Distribuição temporal dos casos de intoxicações por agrotóxicos segundo atividade desenvolvida pelo trabalhador na lavoura do milho, Brasil, 2007-2009 
destaque é que 57 (79\%) intoxicações exógenas ocorreram com o agrotóxico Carbofuran, sendo 53 (59\%) casos confirmados por meio do critério clínico e $5(6 \%)$ pelo critério clínico-laboratorial.

A Figura 4 apresenta o coeficiente de intoxicação por agrotóxicos por município do Brasil, no qual se percebe uma maior incidência de casos no eixo centro-sul do país.

\section{Oportunidade}

Observa-se que 55.507 (94\%) dos atendimentos foram considerados oportunos, uma vez que não ultrapassaram o período máximo, com uma mediana de 0 dias (0-1.055). Tem-se ainda que 66.180 (72\%) das notificações com mediana de 0 dias (0-1.095), 82.055 (95\%) das investigações com mediana de 0 dias (0-731) e 80.675 (96\%) dos encerramentos dos casos com mediana de 1 dia (0-937) foram oportunos.

\section{Estabilidade}

Os recursos humanos direcionados para a vigilância das intoxicações exógenas foram considerados insuficientes por 3 (60\%) técnicos do nível central e 13 (81\%) do nível estadual. Os recursos materiais foram considerados satisfatórios por 5 (100\%) técnicos do nível central e 15 (94\%) do nível estadual. O SINAN NET foi considerado inoperante por 6 (40\%) técnicos estaduais. Observa-se que, os técnicos do nível central estão envolvidos com o sistema de vigilância das intoxicações exógenas em um tempo mediano de 5 meses (3-60 meses). Já os técnicos estaduais apresentaram o dobro desse tempo, com mediana de 1 ano (0-15 anos).

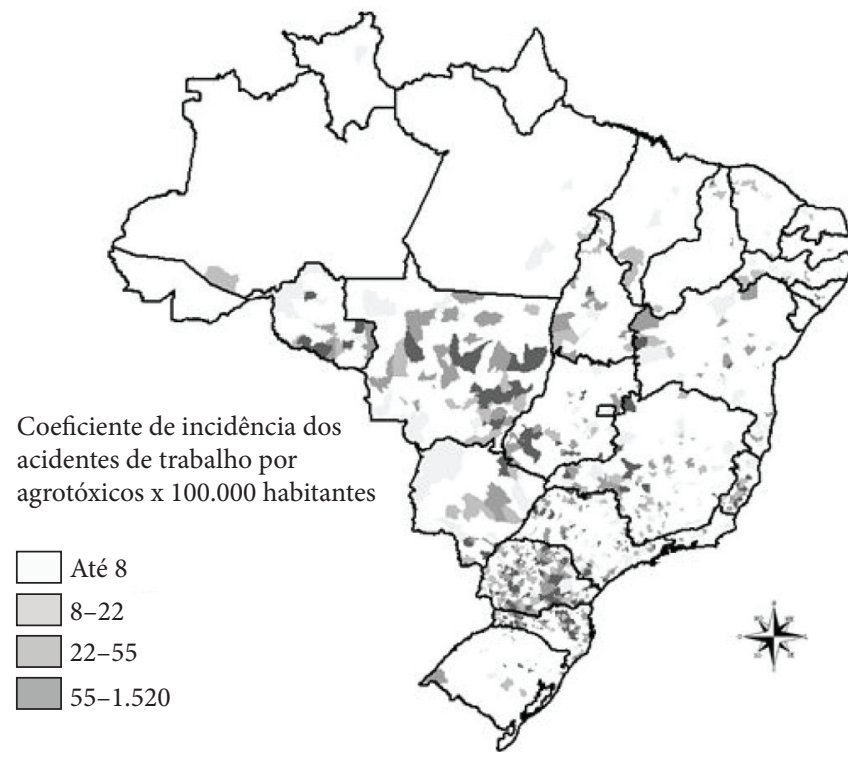

Figura 4. Distribuição espacial dos casos de acidentes de trabalho por intoxicação exógena por agrotóxicos na lavoura do café, Brasil, 2007-2009

\section{DISCUSSÕES E CONCLUSÕES}

Esse sistema de vigilância foi considerado simples, pois os técnicos consideraram o número de variáveis da ficha suficiente e classificaram a obtenção de dados para preenchimento da FII como fácil. É válido destacar que a maioria dos entrevistados não declarou dificuldades para utilizar o SINAN NET e consideraram rápidas as etapas para preencher a FII, incluindo entrada, edição e armazenamento dos dados. Vale ressaltar que o próprio fluxo do sistema, no qual não há interface com outros sistemas ou fontes de informação propicia essa classificação.

O sistema foi considerado flexível, uma vez que em locais onde não existia a rede sentinela da Renast, outros serviços de saúde cumpriram sua função notificadora, flexibilizando essa etapa crítica para o sistema.

O banco das intoxicações exógenas apresentou-se satisfatório quanto à duplicidade de registros, porém, observamos que a completitude da FII foi considerada ruim. O percentual de inconsistência foi elevado na relação entre a "exposição/contaminação decorrente de trabalho" e a "emissão da CAT", pois a CAT deve ser emitida em todo caso de acidente ou doença do trabalho mesmo que não haja necessidade de afastamento ${ }^{18}$. A OMS define intoxicação aguda por agrotóxicos como qualquer doença ou efeito sobre a saúde, resultante de uma exposição suspeita ou confirmada aos pesticidas, que ocorra dentro de 48 horas (com exceção dos raticidas) ${ }^{19}$. Após esse intervalo de tempo, considera-se um caso crônico. Como demonstrado, nem sempre os casos agudos e crônicos foram classificados corretamente. Assim, o sistema de informação das intoxicações exógenas gerou dados de qualidade regular para o período.

A qualidade de dados regular, bem como a prestação de assistência técnica, confecção de boletins epidemiológicos e material educativo apenas por uma minoria dos estados demonstraram uma baixa aceitabilidade do sistema.

A baixa sensibilidade demonstra a fragilidade da vigilância em detectar os casos de intoxicações exógenas que evoluíram para óbito, corroborando as estimativas do Ministério da Saúde de que, para cada evento de intoxicação por agrotóxico notificado, outros 50 não foram notificados ${ }^{20}$.

É válida a manutenção das oficinas de discussão sobre a definição de caso suspeito de intoxicação exógena com o intuito de melhorar a captação de casos conforme vem desenvolvendo o Departamento de Vigilância em Saúde Ambiental e Saúde do Trabalhador. Outras instituições, como a OMS, têm desenvolvido estudos no intuito de padronizar uma definição de caso de intoxicação aguda a fim de melhorar as estimativas de incidência de intoxicações, pois estas exposições costumam ser multiquímicas e, para muitas delas, não há disponibilidade de biomarcadores ${ }^{21}$. 
O VPP foi considerado baixo, devendo-se estabelecer uma definição de caso confirmado e descartado de intoxicação exógena, pois o juízo de valor dos profissionais de saúde foi essencial para a identificação dos padrões clínicos dos pacientes expostos aos agentes frente à diversidade de produtos químicos existentes. Nesse sentido, com o aumento da abrangência da notificação compulsória das intoxicações exógenas torna-se necessário fortalecer o critério laboratorial para confirmação de caso, através da criação de uma rotina de exames laboratoriais capaz de refletir a diversidade química regional à qual as populações estão expostas ${ }^{21}$. Essa rotina deve incluir biomarcadores que indiquem a alteração biológica numa fase precoce, possibilitando a prevenção do dano à saúde, dentre estes marcadores biológicos de efeito, cita-se a determinação da atividade da acetilcolinesterase plasmática e eritrocitária, nas exposições aos pesticidas organofosforados até as alterações citogenéticas decorrentes da intoxicação pelo benzeno ${ }^{6,22}$.

A análise da representatividade através dos dados do SINAN NET e do artigo consultado sobre as mesmas regiões sugeriu que os achados corroboram diversos outros trabalhos que apontam a população masculina com baixa escolaridade como a mais envolvida com a atividade rural, bem como retrata o predomínio da população jovem nesse setor ${ }^{23,24}$. Quanto à variável "situação no mercado de trabalho", observam-se divergências entre as referências consultadas e o SINAN NET, visto que as populações dos estudos são distintas, além disso, a baixa completitude dessa variável no banco de dados compromete a sua análise, bem como as próprias características da agricultura na região, na qual se encontram propriedades pequenas e empresas de diferentes tamanhos, nas quais se concentram trabalhadores com carteira assinada ${ }^{17}$, enquanto em outras regiões tem-se predomínio de agricultura familiar ${ }^{23,30}$.

Porém, observa-se relação entre o aumento dos casos de intoxicação exógena notificados no SINAN NET e o aumento à exposição à folha verde do tabaco no período de colheita da lavoura, destacando que nessa atividade, especificamente no destalo, há maior exposição do trabalhador à folha ${ }^{25,26}$. Não se pode ignorar que um percentual importante desses casos refira-se a uma doença ocupacional, a Doença da Folha Verde do Tabaco (DFVT), na qual a nicotina é absorvida ativamente pela derme e acarreta sinais e sintomas semelhantes aos das intoxicações agudas por agrotóxicos, sendo inclusive um dos diagnósticos diferenciais ${ }^{25,26}$. Desta forma, a inexistência da descrição de sinais e sintomas na FII, bem como de resultados laboratoriais, implicam em uma fundamentação insuficiente para definir o agente tóxico envolvido nessa intoxicação.

$\mathrm{Na}$ lavoura do milho, o incremento dos casos de intoxicações por agrotóxicos no decorrer dos anos pode estar associado ao atual sistema de produção, baseado na utilização do plantio direto sem rotação de culturas e ampliação das épocas de plantio (safra de verão e safrinha), tornando necessário o tratamento de sementes para evitar pragas e garantir uma boa safra ${ }^{27,28}$. Outro destaque são os agrotóxicos mais utilizados na pulverização dessa lavoura: os neonicotinoides e os carbamatos ${ }^{29}$. Seu diagnóstico dá-se por meio de um exame laboratorial de baixo custo, a dosagem da acetilcolinesterase plasmática, capaz de confirmar os casos de forma mais fidedigna do que o critério clínico-epidemiológico ${ }^{6,22,23}$.

A distribuição dos casos de intoxicações exógenas por agrotóxicos registrados no SINAN NET concentram-se nas mesmas direções em que ocorre o uso da terra no Brasil. A lavoura temporária se organiza desde a região Sul do país, estende-se pelo Sudeste, e chega à região Norte através da abertura de frentes de ocupação ${ }^{30}$. A região Nordeste destaca-se pela grande diversidade de usos agrícolas e extrativistas, demonstrando áreas de baixa e elevada incidência de casos de intoxicações ${ }^{30}$. Baseando-se nessas análises, o banco do SINAN NET das intoxicações exógenas apresenta uma representatividade regular para pessoa e excelente para tempo e lugar. Assim, o sistema foi considerado de representatividade regular.

Para o atendimento dos casos de intoxicações exógenas serem considerados oportunos, eles devem ocorrer entre $0 \mathrm{e}$ 48 horas após a exposição do caso ao agente tóxico, uma vez que estudos apontam que a maioria das intoxicações ocorre de forma aguda ${ }^{1,31,32}$. O prazo do atendimento dos casos, sua notificação, investigação epidemiológica e encerramento foram considerados satisfatórios; logo, o sistema foi considerado oportuno.

Os recursos humanos direcionados para a vigilância das intoxicações exógenas foram considerados insuficientes pela maioria dos técnicos dos três níveis administrativos entrevistados. Os recursos materiais foram considerados satisfatórios por grande parte do corpo técnico envolvido na vigilância desse agravo. Também foi analisado o tempo de trabalho nessa vigilância, no qual observamos uma baixa permanência dos técnicos, o que pode sinalizar uma precarização de vínculos de trabalho. Deste modo, o sistema foi considerado instável.

A inexistência de documentos oficiais com descrição dos objetivos do sistema impossibilitou avaliar a utilidade do sistema, seguindo a metodologia adotada.

Podem ter ocorrido dois tipos de vieses: de memória e de informação. O primeiro deve-se ao distanciamento entre o período analisado e a data das entrevistas. $\mathrm{O}$ segundo, porque apenas um estado foi visitado para descrição da relação de fluxo entre estado-município, sendo eleito aquele que possuía o maior número de casos notificados no SINAN NET. Outro viés de informação pode ter ocorrido devido à baixa taxa de 
retorno dos formulários enviados aos técnicos do nível central (39\%) e aos técnicos dos estados (59\%), além de que, entre aqueles que responderam apenas uma minoria dos técnicos atuais trabalhavam na área técnica no período de estudo.

Diante do exposto, evidencia-se que é primordial o aperfeiçoamento do sistema de vigilância das intoxicações exógenas de forma a torná-lo capaz de gerar informações mais fidedignas à realidade, que subsidiem o processo de tomada de decisão no que diz respeito a esse agravo ${ }^{32}$. Assim, recomenda-se ao Departamento de Vigilância em Saúde Ambiental e Saúde do Trabalhador: formalizar métodos específicos para orientar as vigilâncias estaduais e municipais na análise dos dados das intoxicações exógenas; elaborar guia das intoxicações exógenas, contendo objetivos do sistema de vigilância, definição de caso confirmado e caso descartado, tipos de exposição, critérios para confirmação de caso, inclusive exames laboratoriais preconizados, período para coleta oportuna de amostra clínica, além de estabelecer o fluxo do sistema dentro das secretarias estaduais.

\section{AGRADECIMENTOS}

Ao Departamento de Vigilância em Saúde Ambiental e Saúde do Trabalhador, às Coordenações Estaduais do Sistema de Vigilância das Intoxicações Exógenas, à Coordenação Municipal do Sistema de Vigilância das Intoxicações Exógenas de Curitiba. Ao Conselho Nacional de Desenvolvimento Científico e Tecnológico (CNPq) pelo apoio financeiro.

\section{REFERÊNCIAS}

1. Zambolim CM, Oliveira TP, Hoffmann AN, Vilela CEB, Neves D, Anjos FR, et al. Perfil das intoxicações exógenas em um hospital universitário. Rev Med Minas Gerais. 2008;18(1):5-10.

2. Schvartsman C, Schvartsman S. Intoxicações exógenas agudas. J Pediatr (Rio J). 1999;75(Suppl 2):s244-50.

3. Faria NMX, Fassa AGF, Facchini LA. Intoxicação por agrotóxicos no Brasil: os sistemas oficiais de informação e desafios para realização de estudos epidemiológicos. Ciênc Saúde Coletiva. 2007;12(1):25-38.

4. Brasil. Ministério da Saúde. Portaria MS/GM nº 2.325 de 08 de dezembro de 2003. Relação de doenças de notificação compulsória para todo território nacional. Diário Oficial da União 2003; 23 dez.

5. Brasil. Ministério da Saúde. Portaria MS/GM no 33 de 14 de julho de 2005. Inclui doenças à relação de notificação compulsória, define agravos de notificação imediata e a relação dos resultados laboratoriais que devem ser notificados pelos Laboratórios de Referência Nacional ou Regional. Diário Oficial da União 2005; 14 jul.

6. Brasil. Ministério da Saúde. Portaria MS/GM no 777 de 28 de abril de 2004. Procedimentos técnicos para a notificação compulsória de agravos à saúde do trabalhador em rede de serviços sentinela específica no Sistema Único de Saúde - SUS. Diário Oficial da União 2004; 28 abr.

7. Brasil. Ministério da Saúde. Coordenação Técnica de Saúde dos Trabalhadores. Rede Nacional de Atenção Integral à Saúde do Trabalhador. Manual de gestão e gerenciamento. São Paulo: Renast, Mai/2006.

8. Waldman EA. Usos da vigilância e da monitorização em saúde pública. Inf Epidemiol SUS. 1998;7(3):7-26.

9. Romero DE, Cunha CB. Avaliação da qualidade das variáveis epidemiológicas e demográficas do sistema de informações sobre nascidos vivos, 2002. Cad Saúde Pública. 2007;23(3):701-14.

10. German RR, Lee LM, Horan JM, Milstein RL, Pertowski CA, Waller MN, Guidelines Working Group Centers for Disease Control and Prevention (CDC). Updated guidelines for evaluating public health surveillance systems: recommendations from the Guidelines Working Group.. MMWR Recomm Rep. 2001;50(RR-13):1-35; quiz CE1-7.

11. Brasil. Ministério da Saúde. Departamento de Informática do SUS. Sistema de Informação de Agravos de Notificação - SINAN NET: manual do sistema [cited 2010 Oct 12]. Available from: http://www. suvisa.saude.ba.gov.br/informacao_saude/sinan

12. Brasil. Ministério da Saúde. Departamento de Informação e Informática do SUS. DataSUS. Informações de Saúde [cited 2010 Oct 12]. Available from: http://www2.datasus.gov.br/DATASUS/index.php?area=0203

13. Brasil. Ministério do Trabalho e Emprego. Norma Regulamentadora 31 de 3 de março de 2005. Segurança e saúde no trabalho na agricultura, pecuária silvicultura, exploração florestal e aqüicultura. Normas Regulamentadoras de Segurança e Saúde no Trabalho [cited 2011 Jan 10]. Available from: http://portal.mte.gov.br/data/files/8A7C816A36A27 C14013750EE907002CC/NR-31\%20\%28atualizada\%202011\%29.pdf

14. Centro Colaborador da Organização Mundial de Saúde para a Classificação de Doenças em Português. Centro Brasileiro de Classificação de Doenças (CBCD). Classificação Estatística Internacional de Doenças e Problemas Relacionados à Saúde - CID. 10a revisão. São Paulo; 2008 [cited 2010 Oct 12]. Available from: http://www.datasus.gov. $\mathrm{br} / \mathrm{cid} 10 / \mathrm{v} 2008 / \mathrm{cid} 10 . \mathrm{htm}$

15. Instituto Brasileiro de Geografia e Estatística (IBGE). Taxa de desemprego no Brasil [cited 2010 Oct 12]. Available from: http://www.ibge.gov.br/ brasil_em_sintese/tabelas/trabalho_taxa.htm

16. Ministério da Saúde. Portaria MS/GM no 104 de 25 de janeiro de 2011. Terminologias adotadas em legislação nacional, conforme o disposto no Regulamento Sanitário Internacional 2005 (RSI 2005), a relação de doenças, agravos e eventos em saúde pública de notificação compulsória em todo o território nacional. Diário Oficial da União 2011; 25 Jan.

17. Bedor CNG, Ramos LO, Pereira PJ, Rêgo MAV, Pavão AC, Augusto LGS. Vulnerabilidades e situações de riscos relacionados ao uso de agrotóxicos na fruticultura irrigada. Rev Bras Epidemiol. 2009;12(1): 39-49.

18. Brasil. Ministério da Previdência Social. Comunicação de Acidente de Trabalho (CAT) [cited 2010 Dec 02]. Available from: http://www. previdenciasocial.gov.br

19. Thundiyil JG, Stober J, Besbelli N, Pronczuk J. Acute pesticide poisoning: a proposed classification tool. Bull World Health Organ. 2008;86(3):205-9.

20. Silva MV. A utilização de agrotóxicos em lavouras cafeeiras frente ao risco da saúde do trabalhador rural no município de Cacoal/RO (Brasil) [dissertação]. Brasília (DF): Universidade de Brasília; 2006. 
21. Amorim LCA. Os biomarcadores e sua aplicação na avaliação da exposição aos agentes químicos ambientais. Rev Bras Epidemiol. 2003;6(2):158-70

22. Araújo AJ, Lima JS, Moreira JC, Jacob SC, Soares MO, Monteiro MCM, et al. Exposição múltipla a agrotóxicos e efeitos à saúde: estudo transversal em amostra de 102 trabalhadores rurais, Nova Friburgo, RJ. Ciênc Saúde Coletiva. 2007;12(1):115-30.

23. Faria NMX, Rosa JAR, Facchini LA. Intoxicações por agrotóxicos entre trabalhadores rurais de fruticultura, Bento Gonçalves, RS. Rev Saúde Pública. 2009;43(2):335-44.

24. Bartholomay P, Iser BPM, Oliveira PPV, Santos TEHH, Feistler A, Malta DC, et al. Investigação epidemiológica da doença da folha verde do tabaco - região sul do Brasil, dezembro de 2008. In: IX EXPOEPI - Mostra nacional de experiências bem sucedidas em epidemiologia, prevenção e controle de doenças; 2009. Brasília: Ministério da Saúde.

25. Oliveira PPV, Sihler CB, Moura L, Malta DC, Torres MCA, Lima SMCP, et al. Primeiro relato do surto da doença da folha verde do tabaco no Brasil. Cad Saúde Pública. 2010;26(12):2263-9.

26. Costa RV. Fungicidas em milho: aspectos que devem ser considerados no processo de tomada de decisão sobre a aplicação. Jornal Eletrônico da Embrapa Milho e Sorgo. 2008 Jun; 02(08). Sete Lagoas/MG [cited 2011 Jan 26]. Available from: http://www.cnpms.embrapa.br/grao/8_edicao/ grao_em_grao_artigo_01.htm
27. Coelho AM, França GE, Pitta GVE, Alves VMC, Hernani LC. Adubação de milho safrinha [cited 2011 Jan 26]. Available from: http://www. portaldoagronegocio.com.br/conteudo.php?id=22841

28. Milho - qualidade garantida na semente. Revista Rural. 2007 Jul; 113 [cited 2011 Jan 26]. Available from: http://www.revistarural.com.br/ Edicoes/2007/Artigos/rev113_sementes.htm

29. Mota DN, Vieira VCR, Ribas CEDC. Monocultura do café versus diversificação produtiva no assentamento Primeiro do Sul, em Campo do Meio, Minas Gerais: a relação com a segurança alimentar e nutricional sustentável. In: VIII Congreso Latinoamericano de Sociología Rural, Porto de Galinhas; 2010

30. Organização Pan-Americana de Saúde. Organização Mundial de Saúde. Ministério da Saúde. Manual de vigilância da saúde de populações expostas a agrotóxicos. Brasília: OPAS/OMS/MS; 1997.

31. Alonzo HGA. Intoxicações agudas por praguicidas nos centros de toxicologia de seis hospitais universitários do Brasil em 1994 [tese]. Campinas: Universidade Federal de Campinas; 1995 [cited 2010 Oct 12]. Available from: http://www.bibliotecadigital.unicamp.br/document/?co de $=$ vtls000093999\&opt $=4$

32. Jorge MHPM, Laurenti R, Gotlieb SLD. Avaliação dos sistemas de informação em saúde no Brasil. Cad Saúde Colet. 2010;18(1):7-18.

Recebido em: 03/02/2012 Aprovado em: 15/10/2012 